\title{
Análise da pressão em recipientes de plástico devido à reação do ácido acético com bicarbonato de sódio
}

Pressure analyse in plastic containers due to the reaction of acetic acid with sodium bicarbonate

\author{
E.L. Lapolli*1], Sara E. Coelho ${ }^{1}$ \\ ${ }^{1}$ Instituto Federal Catarinense, Abelardo Luz, SC, Brasil.
}

\begin{abstract}
Recebido em 20 de dezembro de 2019. Revisado em 17 de junho de 2020. Aceito em 30 de junho de 2020.
\end{abstract}
\begin{abstract}
Neste artigo, foi desenvolvido uma expressão, a partir de primeiros princípios, para o aumento da pressão provocada pelo dióxido de carbono, resultante da reação entre o bicarbonato de sódio e o vinagre no interior de uma garrafa de polietileno tereftalato (PET). Esta reação, neste tipo de recipiente, é comumente utilizada em competições de lançamento de foguetes no âmbito nacional por estudantes de ensino médio. A motivação dá-se pela segurança, controle e desperdício de material, já que os estudantes realizam os testes sem controle sobre o parâmetros físicos e químicos. Um outro fato, é que as duas equações existentes, descrevem bem o comportamento da pressão em recipientes rígidos fechados até 60 PSI, o qual é metade do valor da pressão de trabalho de uma garrafa PET do tipo reciclável. A expressão, aqui estabelecida, apresenta concordância com a equação empírica até $160 P S I$, e por consequência, com os dados experimentais.
\end{abstract}

Palavras-chave: Bicarbonato, Vinagre, Foguete, Pressão.

In this paper, a theoretical model was developed from first principles to describe the increase the pressure caused by carbon dioxide, formed by the reaction between baking soda and vinegar inside a PET bottle. This reaction, in this type of container, is commonly used in rocket launch in high school students nationwide competitions. The motivation happens by the safety, control and waste of material, since students perform tests without control over physic and chemical parameters. Another fact, is that the two existing theoretical models describe well the pressure behavior in rigid closed containers up until 60 PSI, which is half of the pressure value of a recyclable PET bottle. The model, established here, presents agreement with the empirical model up to 160 PSI and, by consequence, with the experimental data.

Keywords: Bicarbonate, Vinegar, Rockets, Pressure.

\section{Introdução}

\subsection{Educação cientifica}

Ultimamente, construir uma ponte entre o conhecimento de ciências ensinado nas escolas de nível fundamental e médio e o mundo cotidiano dos alunos é um dos grandes desafios. Normalmente, a falta deste vínculo gera distanciamento e marasmo entre os alunos e atinge também os próprios professores. Ao usarem uma abordagem estritamente formal, os professores acabam não explorando as várias possibilidades que existem para tornar a ciência mais "visível"e associá-la com os avanços científicos e tecnológicos atuais que afetam diretamente a nossa sociedade. 1. 2

A educação fundamentada nas inter-relações CiênciaTecnologia-Sociedade (CTS) se revela como alternativa viável para o ensino e a aprendizagem de temas científicos pois supera a simples memorização de regras e fórmulas e insere os discentes em momentos de reflexão capazes de internalizar o significado dos conceitos científicos aplicados a contextos bem definidos. 3 .

Além de proposta inovadora e motivadora as ações embasadas na educação CTS favorecem atitudes de formação pessoal com relação ao ambiente e à qualidade de vida, preparando os estudantes para participarem da sociedade, de forma ativa e crítica, com condições de tomada de decisões considerando aspectos científicos, tecnológicos, econômicos e políticos . [4 5]

Nesse sentido, o professor é o elo entre este indivíduo e o conhecimento científico, cabendo a ele o compromisso ético e moral de desenvolver, no ambiente escolar, o papel de intermediador, de facilitador do conhecimento [3]. Porém, para tornar o assunto abordado interessante ao ponto de vista do aluno atual é necessário que o aluno se sinta desafiado e intrigado com o que lhe é apresentado e essa etapa do processo de ensino-aprendizagem torna-se, mais do que nunca, um dos desafios fundamentais dentro da atividade docente. 6

*Endereço de correspondência: lapollifsc@gmail.com 


\subsection{Motivação}

Dentre os temas de CTS, abordados nos diferentes níveis de estudo, está a astronáutica, cujo objeto central instigador é o foguete, que impressiona a pelas dimensões, tecnologia instalada e no poder de sua decolagem. Muitas pessoas são fascinadas com foguetes, como evidenciam as centenas de milhares de visitantes ao Centro Espacial Kennedy para assistir lançamentos ou visitar o museu. Atualmente, o foguete é um meio necessário para extrapolar a estratosfera. Foi um deste que auxiliou o homem a chegar na lua e explorar Marte com sondas robôs. Os foguetes demonstram princípios básicos importantes de química, astronomia, engenharia e física. Mesmo os lançamentos de foguetes modelos são emocionantes. 33

Muitos professores de física aproveitam isso e usam da construção e do lançamento de foguetes, propelidos à água ou a combustível químico, como um mecanismo de despertar o interesse de estudantes dos ensinos fundamental e médio pela disciplina de Física. Além disso, é um tema com grande potencial estratégico para trabalhos interdisciplinares que, ao mesmo tempo, favorece o ensino de Ciências, podendo ser incorporada ao ambiente escolar. [1-3]

Para os professores, a observação do lançamento dos foguetes pode sugerir curiosidades e, posteriormente, investigações de diversos temas em Física e Química, inclusive para a educação básica, tais como: resistência dos materiais, Leis de Newton, impulso, pressão, centro de massa, leis do movimento, velocidade relativa, balística, gravitação, aerodinâmica, equação de Bernoulli, equação de continuidade, conservação da energia, expansão adiabática de gases, balística, transformações físicas, evidências de reações, combustão, balanceamento, termoquímica, gases, proporção de massas e soluções. [3,7]

Além dos tópicos mencionados acima, os projetos de construção de foguetes expõem os estudantes à excitação do exercício profissional para a carreira de Engenharia Aeroespacial através de alguns conceitos generalistas abordados, como: princípios de aerodinâmica, desempenho, propulsão, estruturas sobre foguetes e mecânica orbital. 3]

Outra contribuição para utilização dos modelos de foguetes, por serem artefatos tecnológicos, são as discussões sobre a Ciência e tecnologia na sociedade, assim como os impactos de um desenvolvimento tecnológico, envolvidos nas etapas de construção, lançamento e aterrissarem dos foguetes e assemelhados. E, ainda, os trabalhos relacionados à construção de foguetes tem proporcionado aos alunos a oportunidade de sociabilizar e popularizar a ciência e suas aplicações de forma lúdica através da divulgação científica, em trabalhos de extensão dirigidos a um público diversificado, como a participação na Mostra Brasileira de Foguetes (MoBFog). [1]

\subsection{Mostra Brasileira de Foguetes (MobFog)}

A MoBFog é um evento que ocorre, anualmente, como uma atividade paralela à Olimpíada Brasileira de Astronomia e Astronáutica (OBA), ambas têm como principal objetivo despertar o interesse e a vocação científica de alunos do ensino básico para a área das ciências, mais especificamente para a astronomia e para a engenharia de foguetes. 7

No que se refere aos alunos do ensino médio, caracterizados como nível IV dentro da MoBFog, o projeto deve englobar a construção de uma base de lançamento e de um foguete. 7 A base de lançamento pode ser confeccionada utilizando-se qualquer tipo de material, enquanto que o foguete deve ser obrigatoriamente construído com garrafas de polietileno tereftalato (PET) de quaisquer capacidades volumétricas. A propulsão do foguete se dá pelo dióxido de carbono $\left(\mathrm{CO}_{2}\right)$ liberado quando se mistura vinagre comercial com bicarbonato de sódio, ou seja, o combustível utilizado por esses protótipos é um dos produtos gerado na reação de neutralização do ácido acético, presente no vinagre, pelo bicarbonato de sódio. [3,7]

A liberação de gás carbônico faz com que o corpo do foguete fique pressurizado. Uma vez pressurizado o sistema, acionando-se o dispositivo de trava do foguete o material residual líquido é expelido propulsionado o foguete para frente. Desta forma, é possível verificar que a pressão interna gerada pelo dióxido de carbono proveniente da reação química, é o principal fator ligado à velocidade de ejeção de massa da cápsula de pressurização e a velocidade de ejeção, por sua vez, determina a velocidade final do foguete no processo de aceleração. Assim, a pressão interna do sistema caracteriza-se como fator fundamental na maximização do alcance horizontal de um foguete desse tipo. 3,7

\subsection{Pressão e segurança}

Há muitos casos em que as competições, locais ou classificatórias para a MoBFog, ocorrem sem controle da segurança e do desperdício de material, já que os estudantes realizam os testes sem controle sobre o parâmetros físicos e químicos. Devemos observar os limites de segurança para a pressão no interior de cada tipo de garrafa utilizada, para que este valor não seja excedido. Isso implica também no entendimento da estéquiometria da reação, pois a proporção, entre bicarbonato e vinagre, deve ser mais próxima do ideal, para que a pressão não seja excedida, e nem haja desperdício dos reagentes.

Existe o problema da reutilização dos foguetes, o que pode acarretar em explosão devido a fadiga, dobras ou arranhões no corpo da garrafa. Há casos em que os competidores utilizam garrafas PET do tipo retornável, pois são dimensionadas para suportar maior stress mecânico, por apresentarem espessura do corpo maior do que a do tipo reciclável. Com isso, pode atingir maiores pressões e reutilizar mais vezes. Em uma consulta a grande rede, 
foi verificado que existem algumas faixas de segurança que devem ser respeitadas conforme segue abaixo.

Para os participantes do fórum do site Instructables, que discute sobre a pressão interna suportada por garrafas plásticas de Coca-Cola, 100 PSI é o limite de trabalho e em pressões internas acima desse valor a probabilidade de explosão é muito grande. Ainda, na discussão é possível encontrar um valor de 13 bar, ou seja, 189 PSI como o maior valor de pressão interna suportada por garrafas plásticas do tipo Coca-Cola 8.

No site AIR COMMAND, uma página especializada em foguetes de garrafa PET propelidos por água pressurizada, são descritos vários testes de explosão de garrafas PET de diferentes capacidades. Foi verificado que uma garrafa de 1,25 $l$ aguenta uma pressão interna de $190 P S I$, já a de $1,5 l$ suporta $175 P S I$ e a de $2,0 l$ 168 PSI. No mesmo site é recomendado que não se ultrapasse $70 \%$ do limite máximo de trabalho como medida de segurança. Assim, neste trabalho foi considerando que o limite máximo para a garrafa PET de $2,0 l$ é de 160 PSI, logo o limite de segurança será de 112 PSI [9].

Em 2012, em ensaios de explosão de garrafas PET, Wilson menciona que o rompimento das garrafas ocorre quando a pressão interna atinge 159 PSI [10]. No mesmo ano Coriolani e colaboradores, realizando testes similares em 12 garrafas PET de Coca-Cola com capacidade de $1,5 l$, verificaram que o limite de pressão interna suportada por esse tipo de garrafa está entre 11,54 bar (147 PSI) e 12 bar (174 PSI). Nesse trabalho os autores não especificaram se as garrafas utilizadas no ensaio são do tipo retornável, uma informação importante visto que garrafas plásticas desse tipo, bem como as de menores capacidades de volumes, apresentam paredes mais espessas sendo capazes de suportar uma maior pressão antes de se romperem 11].

Portanto, obter uma expressão o que estabeleça o comportamento da pressão final em relação a quantidade de reagentes influenciará não só na escolha do melhor material para a construção do foguete como, ter o controle da pressão no interior da garrafa PET se torna essencial na maximização do alcance horizontal do foguete de maneira segura e livre de imprevistos, tais como a abortagem ou explosões.

Consoante com essas idéias, Fonseca e colaboradores mostraram que o comportamento experimental da pressão interna do foguete, ou seja, os valores de pressão encontrados experimentalmente em relação aos valores de volume, temperatura e quantidade de reagentes podem ser previstos de forma satisfatória pela equação de Clapeyron para valores de pressão até 60 PSI. Já considerando comportamento mais próximo de um gás real, a equação de Van der Waals, nessa mesma região, gera valores que se aproximam um pouco mais dos valores experimentais. Porém, nenhuma das duas abordagens permite que a pressão interna seja descrita consistentemente para valores superiores a $60 P S I$ ].
Os autores, ao utilizar a equação de van der Waals, procuram um comportamento de gases mais próximo da realidade, dessa forma, interpretam que a discrepância encontrada não residi nas propriedades físicas do gás, e sim no deslocamento do equilíbrio químico para o lado dos reagentes. Esse deslocamento químico, segundo o princípio de Le Chatelier, é decorrente do aumento da pressão no ambiente da reação, provocando uma diminuição da quantidade de mols de dióxido de carbono gerados na reação química 7 .

Sendo assim, os autores conseguiram estabelecer, de forma empírica, uma equação para a pressão final através da inserção de um fator de atenuação no número de mols de dióxido de carbono na equação dos gases ideais. Através dessa equação, é possível vincular, de forma satisfatória, a pressão interna dos foguetes de garrafa PET à quantidade de reagentes presentes no interior dos foguetes para o regime de valores de pressão de interesse dos lançamentos (80 - 140 PSI) [7].

Nesse sentido, ante o exposto, o presente trabalho vai além. Foi proposto uma nova equação capaz de esclarecer, com maior acurácia, o comportamento da pressão no interior de um recipiente rígido em função da quantidade vinagre utilizada. Para isto, nesse artigo, foi considerado como hipótese, a existência de ar no espaço remanescente, e que o processo termodinâmico é isotérmico. A expressão resultante foi validada via comparação com os valores dos ensaios descrito por Fonseca e colaboradores em 7 .

\section{Modelo considerando mistura de gases}

Para modelar o comportamento da pressão exercida, por um gás liberado em uma reação, no interior de um recipiente, fechado e rígido, utilizamos a teoria de mistura de gases 12].

A equação de estado escolhida foi a de Van der Waals, já que em altas pressões os gases não apresentam comportamento similar ao de um gás ideal, ou seja, em altas pressões. O processo termodinâmico considerado foi o isotérmico, pois apesar da reação ser endotérmica admitimos que o ambiente proverá o calor necessário para manter a temperatura constante. Desta forma, temos uma equação para o estado inicial, com subíndice "o", e outra para o estado final, livre de subíndice. Em ambas equações a temperatura é a mesma, $T_{o}$, a qual vamos admitir como sendo a temperatura ambiente.

$$
\begin{aligned}
\left(P_{o}+a_{o} \frac{n_{o}^{2}}{V_{o}^{2}}\right)\left(V_{o}-b_{o} n_{o}\right) & =n_{o} R T_{o} \\
\left(P+a \frac{n^{2}}{V_{o}^{2}}\right)\left(V_{o}-b n\right) & =n R T_{o}
\end{aligned}
$$

Assim podemos igual as equações (1) e (2) e escrever a pressão final $P$ em função do volume remanescente $V_{o}$

$$
P=\frac{n}{n_{o}}\left(P_{o}+a_{o} \frac{n_{o}^{2}}{V_{o}^{2}}\right)\left(\frac{V_{o}-b_{o} n_{o}}{V_{o}-b n}\right)-a \frac{n^{2}}{V_{o}^{2}}
$$


Neste sentido, o sistema termodinâmico é o volume remanescente $V_{o}$, o qual é a diferença entre o Volume total $\left(V_{T}\right)$ e o volume de vinagre $\left(V_{v}\right)$, ou seja, $V_{o}=V_{T}-V_{v}$. Iremos considera-lo como rígido e aberto, pois todo o gás proveniente da reação irá para o interior deste, produzindo o aumento da pressão. Iremos considerar o valor da pressão do sistema, como sendo o valor da pressão na garrafa pelo teorema de de Pascal.

$\mathrm{Na}$ condição inicial temos somente ar atmosférico no espaço não preenchido pelo vinagre adicionado assim, temos para a co-pressão $a_{o}=19,6925 l^{2} P S I / m_{o l}^{2}$ e o co-volume $b_{o}=0,0322 \mathrm{l} / \mathrm{mol}$. A pressão inicial é a pressão atmosférica $P_{o}=14,6959 P S I$. Lembrando que $R=1,20590 l P S I / K$ mol é a constante universal dos gases. Optou-se por utilizar a unidade se pressão do sistema inglês, PSI (pound square inch) cuja tradução é libra por polegada quadrada. A escolha fundamenta-se no fato que a graduação de grande maioria dos manômetros comerciais está em $P S I$ ou $\mathrm{kgf} / \mathrm{m}^{2}$ ou ambas. Um outro fato, é que os dados disponíveis para comparação estão, também, em PSI.

Após o início da reação as moléculas de gás carbônico produzidas começam a ocupar os interstícios entre as moléculas de ar atmosférico presentes no interior do volume remanescente, ou seja, agora o espaço disponível passa a ser ocupado pela mistura de ar e gás carbônico.
Desta forma, segundo Moran e Shapiro [12], temos que a equação para a co-pressão $(a)$ e o co-volume $(b)$ para a mistura de gases são, respectivamente:

$$
\begin{aligned}
a & =\left(\sqrt{a_{o}} \frac{n_{o}}{n}+\sqrt{a_{c}} \frac{n_{c}}{n}\right)^{2} \\
b & =b_{o} \frac{n_{o}}{n}+b_{c} \frac{n_{c}}{n}
\end{aligned}
$$

Onde, $a_{c}, b_{c}$ e $n_{c}$ são, respectivamente, co-pressão, covolume e número de mols do gás carbônico. Os valores dos coeficientes de Van der Waals para o gás carbônico são: $a_{c}=52,787848 P S I / \mathrm{mol} l^{2}$ e $b_{c}=0,04267 \mathrm{l} / \mathrm{mol}$. O número de mols na mistura é dado por: $n=n_{o}+n_{c}$. E o número de mols de ar atmosférico contidos inicialmente no volume disponível, $V_{o}$, é dado por:

$$
n_{o}=\frac{V_{o} \rho_{a r}}{M_{a r}}=\frac{\left(V_{T}-V_{v}\right) \rho_{a r}}{M_{a r}}=0,04617\left(V_{T}-V_{v}\right)
$$

Para os valores de densidade e massa molar do ar são considerados, respectivamente, $\rho_{a r}=1,2928 \mathrm{~g} / \mathrm{l}$ e $M_{a r}=$ $29 \mathrm{~g} / \mathrm{mol}$.

Substituindo as equações (4) e (5) na equação da pressão da pressão absoluta (3) com os respectivos valores das constantes acima descritos, e em seguida substituindo o resultado, na equação $P_{\text {man }}=P-P_{o}$, obtemos a expressão 7 para a pressão manométrica, em PSI.

$$
P_{\text {mam }}=\frac{8.69 V_{T}^{6}+341.66 V_{T}^{5} V_{v}+4095.11 V_{T}^{4} V_{v}^{2}+9525.51 V_{T}^{3} V_{v}^{3}-60167.27 V_{T}^{2} V_{v}^{4}+73721.54 V_{T} V_{v}^{5}-27525.24 V_{v}^{6}}{0.5912 V_{T}^{6}+14.34 V_{T}^{5} V_{v}+54.43 V_{T}^{4} V_{v}^{3}-376.97 V_{T}^{3} V_{v}^{3}+652.08 V_{T}^{2} V_{v}^{4}-466.21 V_{T} V_{v}^{5}+121.74 V_{v}^{6}}-14,6959
$$

O comportamento da equação é representado pela linha tracejada na figura (1), onde observamos uma concordância com os dados experimentais até 140PSI.

Em uma tentativa de se obter uma equação simplificada em uma estrutura polinomial a partir da equação (7), realizamos uma expansão em série de Taylor desta até a $3^{a}$ ordem em $V_{v}$.

$$
P=105.21 V_{v}+45.6716 V_{v}^{2}+19.668 V_{v}^{3}
$$

Esta equação foi capaz de suprir o comportamento da atenuação da pressão devido a expansão da garrafa até $160 P S I$, conforme linha contínua grossa na figura 1 .

\section{Outros Modelos}

A equação 77 é capaz de modelar o comportamento do aumento da pressão no interior de recipientes fechados e rígidos, ocasionado pela liberação de gás carbônico, devido reação entre bicarbonato e vinagre, na presença de ar no volume remanescente. $\mathrm{O}$ artigo de referência [7] desenvolve duas expressões para descrever o comportamento da pressão. A equação (9) é obtida considerando modelo de Gás ideal, e equação 10 considerando o modelo de Van Der Waals sem levar em consideração mistura.

$$
\begin{aligned}
P & =235,5 \frac{V_{v}}{V_{T}-V_{v}} \\
P & =235,5 \frac{V_{v}}{V_{T}-1,05 V_{v}}-23,5 \frac{V_{v}^{2}}{\left(V_{T}-V_{v}\right)^{2}}
\end{aligned}
$$

Estes equações apresentaram, divergência de valores, em relação aos experimentais, a partir de $60 P S I$, conforme linhas pontilhadas e de traço-ponto, respectivamente, conforme a figura 1. O mesmo autor, na tentativa de melhor descrever o comportamento experimental, de desenvolve um modelo empírico modificando a equação de Clapeyron com segue:

$$
P V=(n-\alpha P) R T
$$

O termo $-\alpha P$ foi inserido intencionalmente, onde o valor de $\alpha$ foi determinado numericamente, via regressão linear, com base nos dados experimentais. O propósito para a inserção ad hoc, foi a suposição de que o aumento da pressão desvia o equilíbrio para o lado dos reagentes, o que conduz a diminuição da quantidade de gás carbônico 


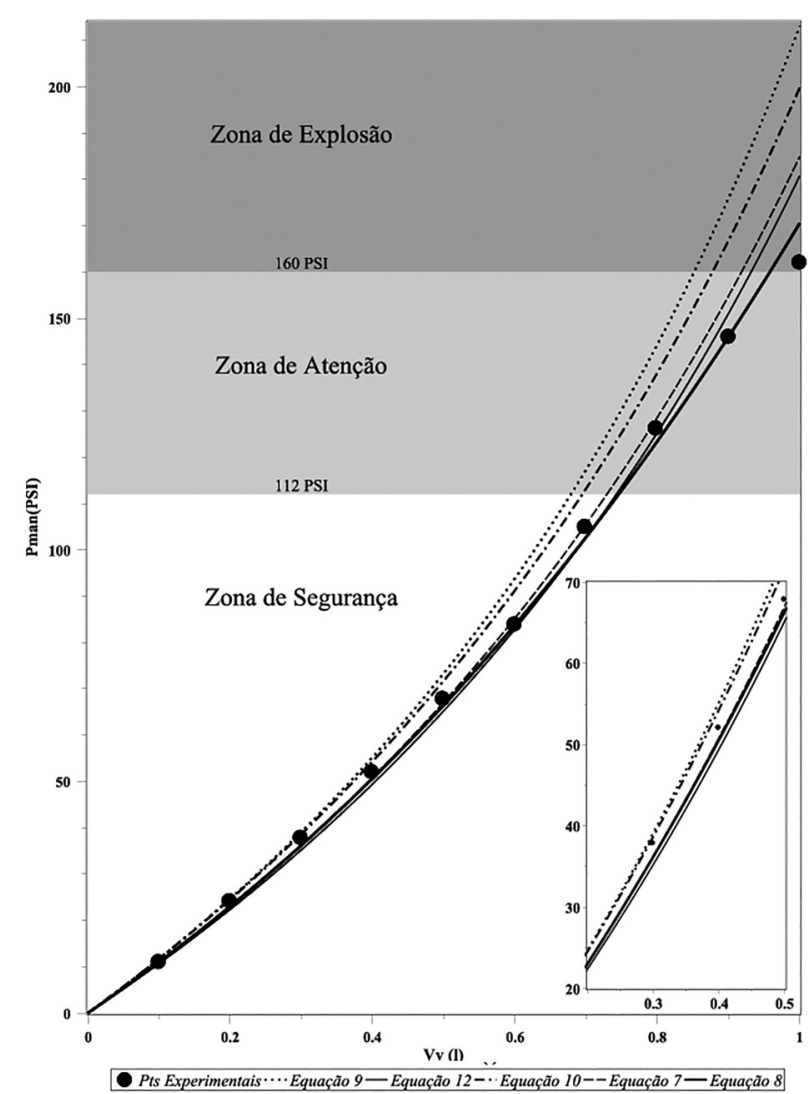

Figura 1: Comparação entre os valores experimentais (círculos) e as equações teóricas (Pontilhada e Traço-Ponto) e empírica (Contínua fina). Os modelos, aqui desenvolvidos, equação 7 e 8 . são representados, respectivamente, pelas linhas tracejada e contínua grossa.

7]. Com estas considerações foi obtido

$$
P=235,5 \frac{V_{v}}{2,3-V_{v}}
$$

O comportamento da equação empírica é representado pela linha contínua fina na figura 1

\section{Resultados e discussões}

Ao avaliar as hipóteses elaboradas no artigo de referência [7], verifica-se algumas inconsistências que acabaram ocasionando nas divergências, a partir de 60 PSI, entre as equações (9) e (10) e os dados experimentais (figura 11. A primeira, é que os autores supõem um processo adiabático, porém, em nenhum momento esta hipótese foi abordada no equacionamneto. Uma outra, é que a temperatura do sistema foi considerada como sendo a do ambiente, logo os valores das pressões são para a referida temperatura, o que não pode o correr, já que ao considerar o processo como adiabático, o qual é um tipo de processo politrópico, ou seja, todas as coordenadas termodinâmicas variam, logo, deveria existir uma temperatura referente a pressão final. Devido a reação ser endotérmica, parte do calor do sistema $V_{o}$ é perdido para o produto da reação, desta forma este se torna diatérmico, logo, não deveria ser considerado adiabático. Um outro fato, é que os autores não levaram em consideração a presença de ar no volume remanescente no sistema, já que esta quantidade influencia no resultado final.

As expressões no artigo de referência não representarem bem o comportamento para pressões a cima de $60 P S I$, Fonseca [7] propõe que esta divergência reside no fato que a reação não ocorre na integra, pois o equilíbrio o desloca para o lado dos reagentes, devido ao aumento da pressão (Princípio de Le Chatelier). Ao fazer esta consideração, o autor despreza os efeitos físicos, e elabora um modelo empírico fundamentado em sua justificativa química. Desta forma, a equação (12) é uma equação empírica, não tem origem em primeiros princípios, e sim em uma suposição do autor fundamentado no princípio de Le Chatelier, serve para descrever o comportamento de dados experimentais dentro de uma certa faixa de valores, pois o parâmetro $\alpha$ é ajustado por meio de dados experimentais.

Considerando uma garrafa PET de 2litros, podemos observar que, para o regime de pressão, adequados para lançamentos de foguetes de garrafa PET, a equação (7) gera valores que estão de acordo com os valores experimentais [7], sendo, então, capaz de modelar o comportamento experimental, como mostrados na figura (1). Por consequência, está em concordância com os valores gerados pela equação empírica (12) [7]. Os valores dos pontos experimentais foram extraídos com o auxílio do programa WebPlotDigitizer na versão online, a partir da imagem do gráfico publicado no artigo de referencia 7 .

A equação (8) tem a intenção de tentar predizer, de forma mais próxima do real, o que acontece no interior de uma garrafa PET durante a reação, porém, este não é um recipiente rígido, o que leva a divergências para pressões acima de 140PSI. Pressupomos que estas divergências são ocasionadas pela deformação da garrafa, ou seja pelo aumento do volume total, tal como um balão.

A equação (8), a qual é uma aproximação serie de Taylor para a equação (7), é capaz de suprir o comportamento da atenuação da pressão devido a expansão da garrafa até $160 P S I$, figura 1 . Assim é uma equação simplificada que pode ser utilizada para prever a pressão em garrafas de $2 l$.

Ao considerarmos que o volume livre da garrafa está previamente preenchido por ar atmosférico à temperatura ambiente, teríamos um número adicional de moléculas de gás confinadas no volume remanescente (sistema) $V_{o}$. Com estas considerações, foi possível obter um modelo teórico que descrevesse o comportamento da pressão.

A partir dos dados experimentais, foi obtido um polinômio de terceiro grau via método de mínimos quadrados. Ao desprezar o último ponto experimental, o de maior pressão, nós obtemos a seguinte expressão empírica.

$$
P=109.450 V_{v}+48.391 V_{v}^{2}+12.906 V_{v}^{3}
$$


Realizando uma comparação visual entre os valores dos coeficientes de cada equação, averiguou-se a proximidade entre eles.

\section{Conclusão}

As equações 7 e 8 , proposta neste artigo, transcrevem muito bem, o comportamento do da mistura ar e gás carbônico, até o limite de segurança, do recipiente do tipo reciclável de $2 l$. Também que a hipóteses levantadas foram sustentadas quando realizada as devidas comparação com os dados experimentais, ou seja, o processo é isotérmico e que o ar presente influencia na evolução da pressão e que o ultimo ponto experimental sofre atenuação devido a expansão do recipiente.

A equação 8 é uma equação polinomial simples, que pode ser amplamente ser utilizada para compreensão do comportamento do aumento da pressão em função mistura entre vinagre e bicarbonato de sódio, dando assim, uma maior segurança aos competidores da MoBFog.

Para recipientes do tipo retornável, ainda requer um estudo experimental para validar nossos resultados acima de $160 P S I$, já que estes podem chegar a uma pressão de $300 P S I$ quando reforçados externamente, o que tornao mais próximo de um recipiente rígido. Neste sentido, poderíamos avaliar se o deslocamento do equilíbrio, em altas pressões, realmente ocorre.

\section{Considerações finais}

Com este trabalho, acreditamos que estaremos contribuindo com os alunos do ensino médio, que visam participar da MoBFog, a desenvolverem seus projetos com mais controle, sobre os parâmetros determinantes na maximização do alcance horizontal dos seus foguetes com mais segurança.

Para complementar, há equipes que aquecem o vinagre, com a falsa impressão de que irá ocorrer uma maior liberação de gás carbônico durante a reação com o bicarbonato de sódio. A temperatura não influencia na estéquiometria do processo, e sim na velocidade de reação e no aumento de liberação de vapor. Em nosso trabalho a temperatura não aparece no equacionamneto devido a suposição de que o processo é isotérmico, e como verificado, foi uma boa aproximação.

\section{Referências}

[1] E.C. Valadares, Quím. Nova na Esc. 13, 38 (2001).

[2] J.N. Silva, J.S. Amorim, L.P. Monteiro e K.H.G. Freitas, Scientia Plena 13, 012701 (2017).

[3] P.V.T. Souza, N.Q. Amauro e M. Fernandes-Sobrinho, Quím. Nova na Esc. 3, 186 (2018).

[4] L. Martínez e A. Rojas, TED 19, 44 (2006).

[5] W.L.P. Santos, in: CTS e educação cientí-fica: desafios, tendências e resultados de pesquisa, editado por W.P.
Santos e D. Auler (Editora Universidade de Brasília, Brasília, 2011).

[6] A.C.P. Fernandes, L.T.S. Auler, J.A.O. Huguenin e W.F. Balthazar, Rev. Bras. Ensino Fís. 38, e3504 (2016).

[7] M.V.F. Snovarski, I.M.F. Rodrigues e M.B.F. Snovarski, Rev. Bras. Ensino Fís. 40, e3504 (2018).

[8] https://www.instructables.com/community/how-muchpsi-does-a-coke-bottle2l-hold/, acessado em 24/05/2018.

[9] AIR COMMAND, Whater Rocket, disponível em http://www.aircommandrockets.com/procedures.htm, acessado em 24/05/2018

[10] https://www.youtube.com/watch?v=Kiu79vt7D2M, acessado em 24/05/2018.

[11] C. Coriolani, F. Licciardello e G. Muratore, Italian Journal of Food Science 24, 170 (2012).

[12] M.J. Moran e H.N. Shapiro, Princípios de Termodinâmica para Engenharia (LTC Editora, São Paulo, 2006), $5^{\mathrm{a}}$ ed. 